\title{
Continuous instinct control for powered knee-ankle prostheses
}

\author{
Kaixin Shen ${ }^{1 *}$, Qing $\mathrm{Wei}^{1}$, Yongshang Huang ${ }^{1}$, and Hongxu $\mathrm{Ma}^{1}$ \\ ${ }^{1}$ Robotics Research Center, College of Intelligence Science and Technology, National University of \\ Defense Technology, China
}

Keywords: Powered knee-ankle prostheses, Virtual constraint, Phase variable, Instinct, Continuous.

\begin{abstract}
In order to improve the life quality of lower extremity amputees, many researchers have studied the powered knee-ankle prosthesis. Various parameters must necessarily be adjusted for the finite state machine impedance model method. Hybrid zero-dynamic (HZD) assumptions are ideal, and with this method measurement information of existing sensors can be limited. The virtual constraint method offers better comprehensive performance at present and can realize the continuous control for the whole gait cycle. The problem with virtual constraint is mainly the selection of phase variables. The joint trajectory of the virtual constraint is derived from a healthy individual, but the joint trajectory of the amputee's normal walking is difficult to obtain. In response to the above problems, this paper proposes an instinctive human joint trajectory, selecting the phase variable associated with the hip joint angle and angular velocity. The Fourier transform solves the expression of the joint trajectory, and the virtual constraint unifies the control method of the entire gait cycle. The simulation results prove the feasibility of the scheme.
\end{abstract}

\section{Introduction}

According to the relevant literature and the data of the Amputee Coalition and the Stroke Center $[1,2,3]$, it is clear that the problems of restoring normal life functions for the lower extremity amputee continue to require additional study. Past passive prostheses are uncomfortable, have no safety measures, require a great deal of physical energy on the part of the wearer, and provide poor human-computer interaction. A large number of researchers have researched the shortcomings of passive prostheses and many problems have been well resolved. Now the power knee prosthesis technology is relatively mature, but many shortcomings remain.

The finite state machine impedance control method divides the human gait into four stages: early stance period, late stance period, early swing period, and late swing period [7]. There are three parameters for each state to be adjusted in the impedance model $[5,6]$. There

\footnotetext{
*Corresponding author: 1654346091@qq.com
} 
are a total of 12 parameters for each specific mode of motion to be adjusted. The human locomotion mode mainly includes walking at different speed levels, walking up and down stairs, up and down slopes, and conversion between various modes. Therefore, various parameters inevitably need to be adjusted for the finite state machine impedance control scheme. The adjustment of each parameter takes a great deal of time from a professional technician.

A prosthesis based on HZD control assumes that the bipedal model is in contact with the ground on only one leg stance $[8,9,10]$, i.e the gait stance period and the swing period respectively account for $50 \%$ of gait cycle. However, the human actual movement is $60 \%$ in the stance period and $40 \%$ in the swing period. HZD divides the human gait cycle into: the prosthesis support period, the impact of the prosthesis support to the healthy side support, the healthy side support period, and the impact of the healthy side support to the prosthesis support. HZD assumes that the impact periods before and after the joint angle are equal and the feedback linearization control is applied. However, the prosthesis and the human residual limb are connected by a socket, and the bipedal information obtained by the prosthesis is limited, so the difference between the amputation and the healthy side produces two different gait cycles.

The key problem of the virtual constraint is the selection of the phase variable and the method of parameterizing the joint trajectory on the unified control of the powered knee-ankle prosthesis. Selecting the variable associated with the hip joint as a phase variable can achieve uniform control of the entire gait cycle. The study in [12] shows that the Unified Phase Variables of Relative Degree Two in Phase Hip Velocity and Phase Hip Integral and Sagittal and Frontal Hip Angle is the least affected by noise and best utility and Phase Hip Integral can be controlled by PD. However, the system order is increased by one. The method of parameterizing the joint trajectory by the phase variable is mainly the Bezier curve and the discrete Fourier transform.

For existing virtual constraint control $[11,13,14,15]$, the lower limb joint trajectory is derived from healthy individuals. However, there are still some differences between the joint trajectory of healthy individuals and amputees. In the absence of an emergency, the fixed movement pattern of a person is an instinctive reaction that does not require real-time brain control. The paper proposes the CCI interface adjusting the joint trajectory [16].Therefore, this paper proposes an instinctive human joint trajectory. The joint trajectory is obtained by a Bezier piecewise fitting using the joint trajectory of a healthy individual. This process can adjust the shape of the curve depending on demand. Then, the joint trajectory is expressed using a Fourier transform. Finally, a virtual restraint control method is used to control the powered knee-ankle prosthesis. The joint trajectory output from the virtual restraint control system is analyzed and the patient's opinion is sought. Then, the above process is repeated until a patient's most comfortable joint trajectory is produced. A simulation model is used for verification. Using virtual constraints greatly reduces the parameters of the required adjustments. A Bezier spline interpolation curve with an adjustable joint trajectory specific position shape ensures a more complete sequence of true joint trajectories. The virtual constraint system uses discrete Fourier transform methods to ensure the periodic continuity of the joint trajectory.

This paper is organized as follows. Section 2 describes the model and methods for a transfemoral amputee. Section 3 details the simulation and result analysis. Section 4 presents the discussion and conclusion. Section 5 lists acknowledgments, followed by references.

\section{Model and method}




\subsection{Model}

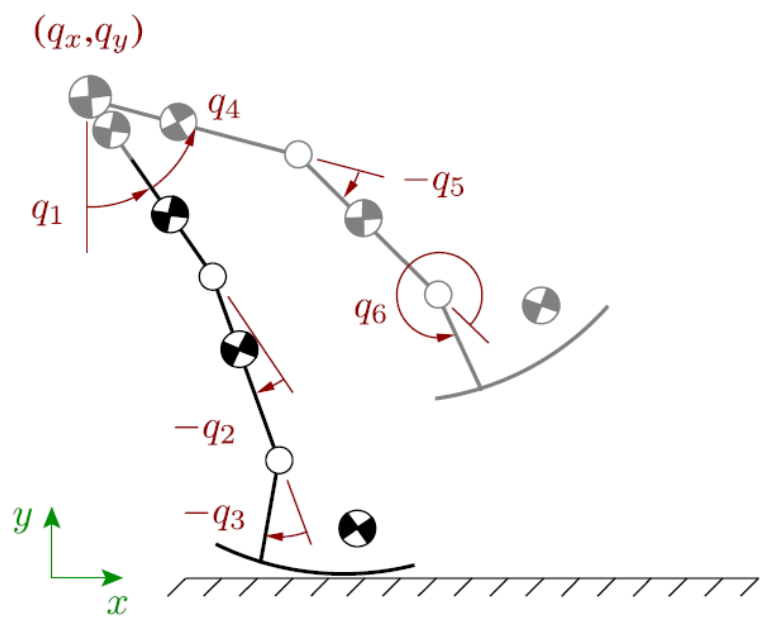

Fig. 1. Schematic of the unilateral, transfemoral amputee model (reproduced from [8]).

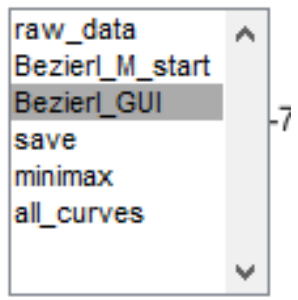

Fig. 2. GUI Button.

Located in the lower left corner of the GUI interface. Clicking raw_data, raw data curve appears on the GUI interface. Clicking BezierI_GUI indicates that the Bezier curve fitting is completed. Clicking BezierI_M_start shows you how to fit the desired curve according to the actual situation. Clicking "save", the data of the desired curve will be saved in the current folder using .mat format. Clicking "minimax" shows the extreme points of the Bezier curve in the GUI interface. Clicking all_curves shows all curves in the GUI interface.

The unilateral and transfemoral amputee model is shown in Figure 1., from the literature [8]. The prosthesis is connected to the thigh of the residual limb by a socket. The point $\left(q_{x}, q_{y}\right)$ represents the Cartesian coordinates of the hip joint. , . $q_{1}$ is an underactuated hip joint angle. $q_{2}, q_{3}$ is the driving joint angle of the prosthesis. $q_{4}, q_{5}, q_{6}$ is the relative angle of the drive joint of the healthy side leg.

\subsection{Method}

B-spline interpolation GUI interface.

The B-spline algorithm is represented as follows. 


$$
q\left(t_{i}\right)=\sum_{k=0}^{m}\left(\begin{array}{c}
m \\
k
\end{array}\right) P_{k}\left(1-t_{i}\right)^{m-k} t_{i}^{k}, 0 \leqslant t_{i} \leqslant 1
$$

where $q\left(t_{i}\right)$ is an interpolated point at parameter value $t_{i}, m$ is degree of Bezier curve and $P_{k}$ is $K^{t h}$ control point. To generate $n$ points ( $n$ is count of interpolating points) between first and last control points inclusive, the parameter $t_{i}$ is uniformly divided into $n-1$ intervals between 0 and 1 inclusive. This article uses a B curve degree of 3 .

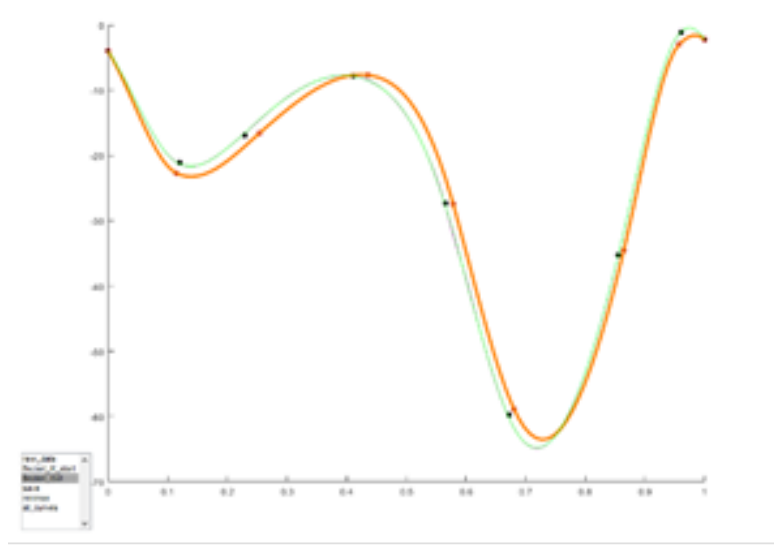

Fig. 3. Expected joint trajectory adjustment picture.

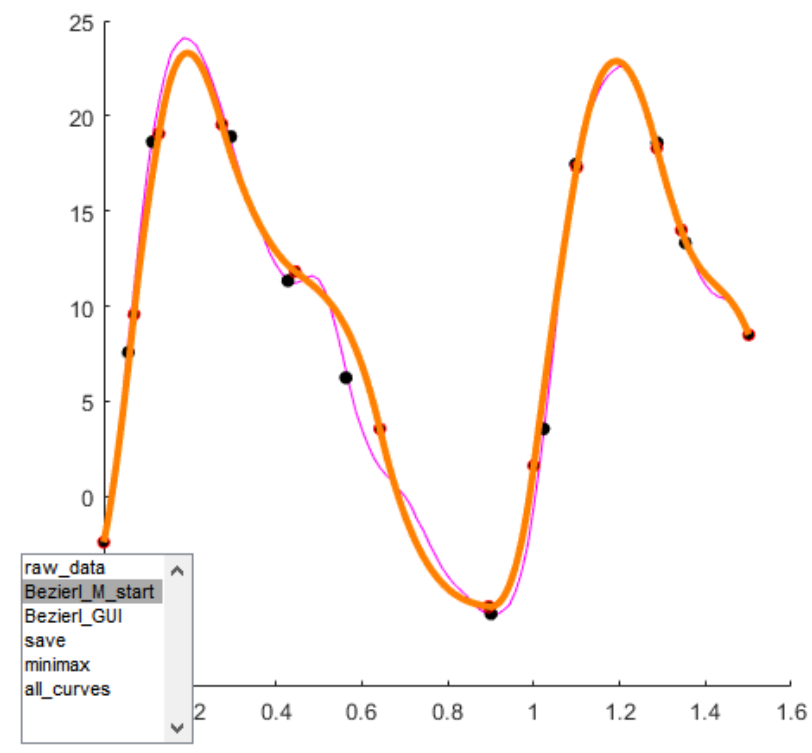

Fig. 4. Desired hip trajectory.

Figure 2 shows the GUI operation button. Clicking the corresponding button in the diagram from top to bottom will produce the relevant curve in the graph window. Figure 3 
shows the joint trajectory, where the green curve generally represents the joint trajectory of a healthy individual, the orange curve represents the joint trajectory to be adjusted, and the black dot represents the control point. When a part of the joint trajectory needs to be adjusted, click on the control point closest to it. Click on the "save" button to export the desired joint trajectory sequence.

Selecting the phase variable in [13] parameterizes the joint trajectory. At this point, the parameterization process uses the Fourier transform [11]. Finally, the prosthesis is controlled according to the virtual constraint prosthetic controller structure [15]. Virtual constraints can be expressed by

$$
y_{i}=q_{i}-h_{i}^{d}\left(s_{h}\right)
$$

where $q_{i}$ is the measured angular position of joint $i$ (with $i=k$ for the knee or $i=a$ for the ankle), and $h_{i}^{d}$ is the desired joint angle trajectory as a function of the normalized phase variable $s_{h} \in[0,1)$.

Output PD controllers typically have the form

$$
\tau_{d i}=-K_{p i} y_{i}-K_{d i} \dot{y}_{i}
$$

where $K_{p i}>0$ is the proportional gain affecting the stiffness of joint $i$ about its angular trajectory, and $K_{d i}>0$ is the derivative gain correcting velocity tracking error $\dot{y}_{i}$.

\section{Simulation and results}

For the purposes of this study, a unilateral, humeral amputation model with a height of $165 \mathrm{~cm}$, a weight of $56.7 \mathrm{~kg}$, a trunk mass of $32.773 \mathrm{~kg}$, a thigh mass of $5.67 \mathrm{~kg}$, a shank mass of $2.64 \mathrm{~kg}$, a thigh length of $31.4 \mathrm{~cm}$, and a shank length of $45.5 \mathrm{~cm}$ was simulated. The calculation method of each part mass and length and the original reference trajectory of each joint for healthy individuals are derived from [17]. These simulations were derived using matlab simulation.

In Fig. 3, the orange curve represents the hip joint trajectory. Human body movement is mainly symmetrical, so the left and right hip joints take the same trajectory. Fig. 5 The green curve represents the contralateral knee trajectory and the orange represents the trajectory that will be used for the prosthetic knee joint. Fig. 6 indicates the ankle trajectory. During the movement of the prosthesis, the ankle trajectory changes little, so the change of the knee joint is mainly considered. The phase plan is produced using the thigh angle and angular velocity is shown in the Fig. 7. It can be reported that the phase variable satisfies monotonicity and boundness and the residual legs are under-actuated. 


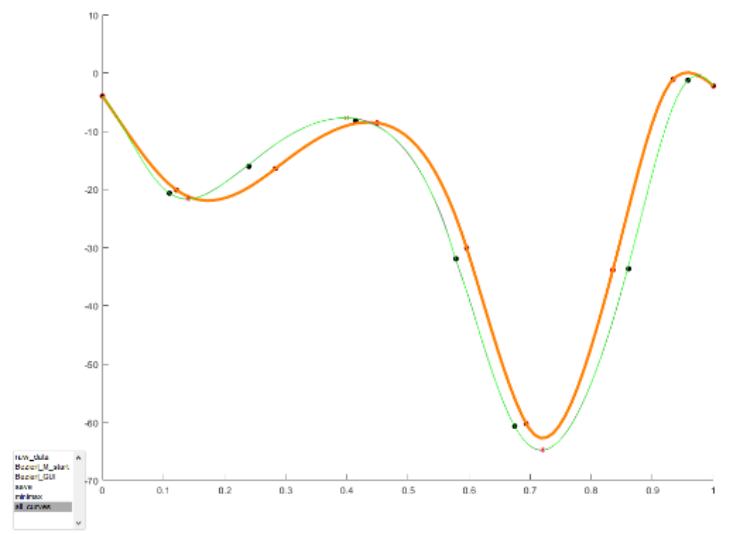

Fig. 5. Desired knee trajectory.

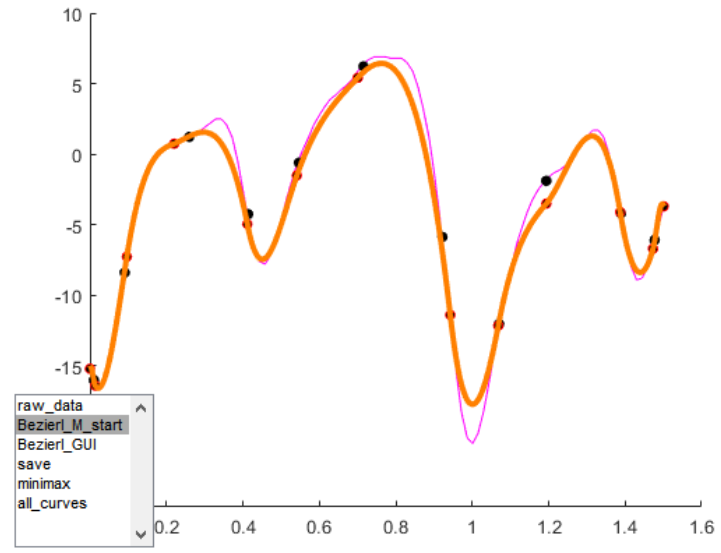

Fig. 6. Desired ankle trajectory.

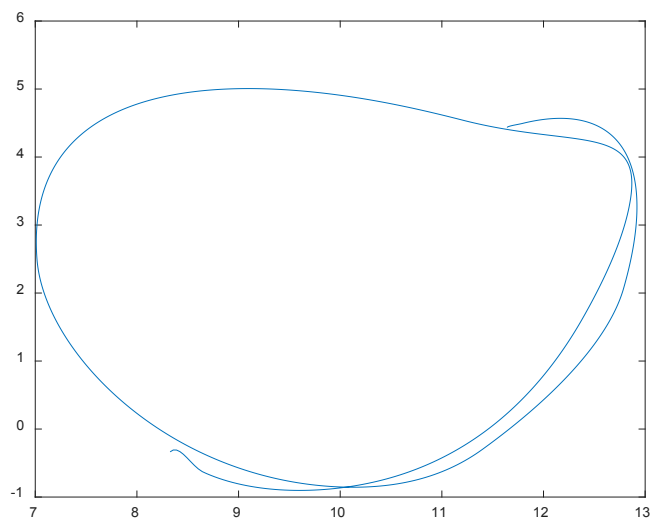

Fig. 7. phase plane. 
Then, the Fourier transform is applied to solve the joint trajectory of the phase variable parameterization. Finally, the joint trajectory is applied to the prosthetic knee. After constantly repeated adjustments, the final knee trajectory of powered knee-ankle prosthesis is shown in the Fig. 5. The yellow solid line indicates the given desired trajectory and the solid blue line indicates the actual trajectory produced by the prosthetic knee joint. The joint trajectory of the ankle joint is shown in Fig. 6. The yellow solid line indicates the given desired trajectory, and the solid blue line indicates the actual trajectory produced by the prosthetic ankle joint. According to the joint trajectory of the final dynamic knee prosthesis, Fig. 8 and Fig. 9 demonstrate that the method proposed in this paper can achieve superior walking performance.

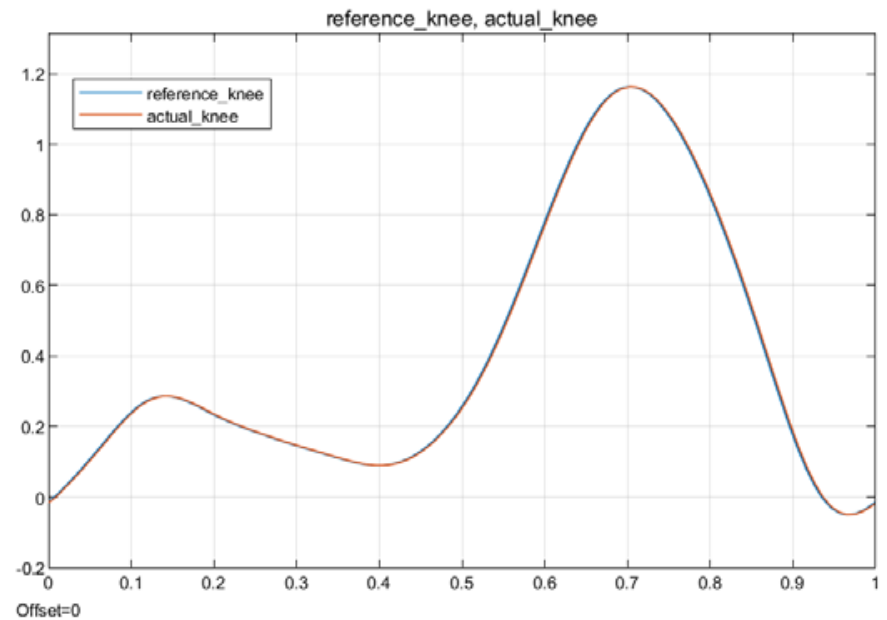

Fig. 8. Knee joint experiment results comparison picture. The yellow solid line indicates the given desired trajectory and the solid blue line indicates the actual trajectory produced by the prosthetic knee joint.

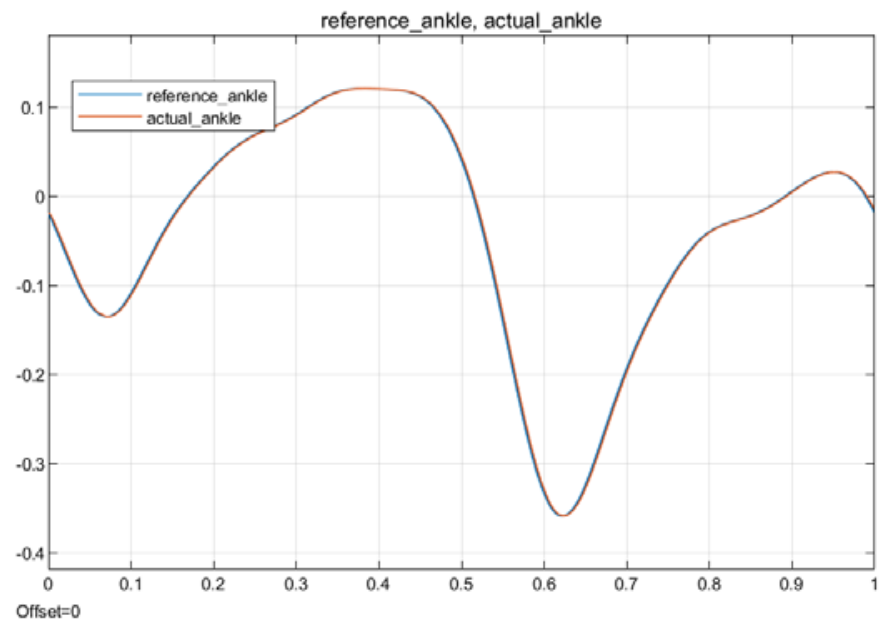

Fig. 9. Ankle joint experiment results comparison picture. The yellow solid line indicates the given desired trajectory, and the solid blue line indicates the actual trajectory produced by the prosthetic ankle joint. 


\section{Discussion and conclusion}

The finite state machine impedance model method requires numerous parameter adjustments. Virtual constraints greatly reduce the parameters that need to be adjusted. The phase variable associated with the hip joint angle ensures that the motion path is independent of time, because time has been continuously changing. But a continuously changing joint trajectory is extremely dangerous. Selecting a phase variable that is continuous and monotonous throughout the gait cycle parameterizes the joint trajectory, ensuring uniform control over the gait cycle. The Fourier transform guarantees periodic continuity in the control process. The B-spline interpolation GUI interface, which can locally adjust the joint trajectory, reduces the time to adjust parameters and measure data. Simulation results demonstrate the feasibility of the proposed method. However, because the simulation environment is ideal, and the density of each part of the human body is assumed to be uniform, the ground environment is its own structure, etc. Therefore, the actual power knee prosthesis will have many problems. This method will be applied to the actual powered knee prosthesis in the future.

Thanks to the help of teachers and classmates. The project is National Key R\&D Program of China, numbered 2018YFC2001300. Topic 4 "Development of Man-Machine Cooperative Control System in Multi-Motion Mode".

\section{References}

1. Ziegler-Graham K, MacKenzie E J, Ephraim P L, et al. Estimating the prevalence of limb loss in the United States: 2005 to $2050[\mathrm{~J}]$. Archives of physical medicine and rehabilitation, 2008, 89(3): 422-429.

2. Amputee Coalition (2008). [Online]. Available: http://www.amputee-coalition.org

3. Stroke Center (2008). [Online]. Available: http://www.strokecenter.org

4. Villarreal D J, Gregg R D. A survey of phase variable candidates of human locomotion[C]//2014 36th Annual International Conference of the IEEE Engineering in Medicine and Biology Society. IEEE, 2014: 4017-4021.

5. Lawson-Brian E., Goldfarb-Michael, IMPEDANCE \& ADMITTANCE -BASED COORDINATION CONTROL FOR ROBOTIC LOWER LIMB, MECHANICAL ENGINEERING, vol: 136, pp: 62-67, 2014.

6. Lawson B E, Mitchell J, Truex D, et al. A robotic leg prosthesis: Design, control, and implementation[J]. IEEE Robotics \& Automation Magazine, 2014, 21(4): 70-81.

7. Simon A M, Ingraham K A, Fey N P, et al. Configuring a powered knee and ankle prosthesis for transfemoral amputees within five specific ambulation modes[J]. PloS one, 2014, 9(6): e99387.

8. Martin A E, Gregg R D. Hybrid invariance and stability of a feedback linearizing controller for powered prostheses[C]. American Control Conference (ACC). IEEE, 2015: 4670-4676.

9. Gregg R D, Martin A E. Prosthetic leg control in the nullspace of human interaction[C]//2016 American Control Conference (ACC). IEEE, 2016: 4814-4821.

10. Martin A E, Gregg R D. Stable, robust hybrid zero dynamics control of powered lower-limb prostheses[J]. IEEE transactions on automatic control, 2017, 62(8): 3930-3942. 
11. Quintero D, Martin A E, Gregg R D. Unifying the gait cycle in the control of a powered prosthetic leg[C]. IEEE International Conference on Rehabilitation Robotics (ICORR). IEEE, 2015: 289-294.

12. Villarreal D J, Gregg R D. Unified phase variables of relative degree two for human locomotion[C]. 38th Annual International Conference of the IEEE Engineering in Medicine and Biology Society (EMBC). IEEE, 2016: 6262-6267.

13. Quintero D, Lambert D J, Villarreal D J, et al. Real-time continuous gait phase and speed estimation from a single sensor[C]//2017 IEEE Conference on Control Technology and Applications (CCTA). IEEE, 2017: 847-852.

14. Villarreal D J, Poonawala H A, Gregg R D. A robust parameterization of human gait patterns across phase-shifting perturbations[J]. IEEE Transactions on Neural Systems and Rehabilitation Engineering, 2016, 25(3): 265-278.

15. Quintero D, Villarreal D J, Lambert D J, et al. Continuous-phase control of a powered knee-ankle prosthesis: Amputee experiments across speeds and inclines[J]. IEEE Transactions on Robotics, 2018, 34(3): 686-701.

16. Quintero D, Reznick E, Lambert D J, et al. Intuitive Clinician Control Interface for a Powered Knee-Ankle Prosthesis: A Case Study[J]. IEEE journal of translational engineering in health and medicine, 2018, 6: 1-9.

17. DAVID A.WINTER. JOHN WILEY \& SONS, INC, WILEY, Reference to a book: BIOMECHANICS AND MOTOR CONTROL OF HUMAN MOVEMENT, Fourth Edition. 